Artículo

\title{
Bioestimulantes en la calidad de frutos de chile habanero
}

\author{
Félix David Murillo-Cuevas ${ }^{1}$ \\ Héctor Cabrera-Mireles ${ }^{2 \S}$ \\ Jacel Adame-García ${ }^{1}$ \\ Andrés Vásquez-Hernández ${ }^{2}$ \\ Adrián de Jesús Martínez-García ${ }^{1}$ \\ Rebeca Luria Moctezuma ${ }^{1}$ \\ ${ }^{1}$ Tecnológico Nacional de México-Úrsulo Galván. Carretera Cd. Cardel-Chachalacas km 4.5, Úrsulo \\ Galván, Veracruz, México. CP. 91667. ${ }^{2}$ Campo Experimental Cotaxtla-INIFAP. Carretera Federal \\ Veracruz-Córdoba km 34.5, Medellín de Bravo, Veracruz, México. CP. 94992. \\ ${ }^{\S}$ Autor para correspondencia: cabo.cabrera50@gmail.com.
}

\section{Resumen}

La producción de chile habanero se realiza principalmente con fertilización química, la cual puede ser ineficiente, ya que gran parte del fertilizante aplicado se libera al medio ambiente y a menudo puede dejar de estar disponibles para las plantas. Una forma en la que se puede reducir el uso de fertilizantes químicos y mejorar la absorción de nutrientes por parte del cultivo es mediante el uso de bioestimulantes en la fertilización de las plantas. El objetivo del trabajo fue evaluar tres bioestimulantes microbianos sobre semillas, plántulas y la calidad de fruto de chile habanero bajo condiciones protegidas de macrotúnel. El trabajo se realizó en el año 2021 en el Tecnológico Nacional de México, Campus Úrsulo Galván. Las semillas de chile habanero utilizadas fueron de la variedad Jaguar proporcionadas por el Campo Experimental Cotaxtla-Instituto Nacional de Investigaciones Forestales, Agrícolas y Pecuarias. Los tratamientos que se evaluaron fueron: 1) $\mathrm{T}_{2} 2^{\circledR}+$ micorriza INIFAP ${ }^{\circledR}$; 2) Mix $^{\circledR}$; 3) Genifix ${ }^{\circledR}$; y 4) testigo. Se realizó una evaluación de los tratamientos en germinación de semillas, crecimiento y biomasa de plántulas y calidad de frutos de chile habanero. No se registraron diferencias significativas en germinación de semillas, pero si en altura y peso seco de plántulas a los 20 días después de la inoculación, ya que las semillas inoculadas con el bioestimulante Genifix fueron las que alcanzaron la mayor altura y peso seco. En cuanto al tamaño y peso de frutos, las plantas tratadas con los bioestimulantes produjeron frutos significativamente más grandes y pesados que las plantas testigo.

Palabras claves: Bacillus, Trichoderma, biofertilizantes, hortalizas.

Recibido: agosto de 2021

Aceptado: octubre de 2021 


\section{Introducción}

Actualmente el uso de fertilizantes químicos en la agricultura es muy ineficiente; gran parte del fertilizante aplicado se libera al medio ambiente, se lavan del suelo por la escorrentía y a menudo pueden dejar de estar disponibles para las plantas; a través, de una transformación química, física o biológica (Sánchez et al., 2001; Daverede et al., 2004). De tal forma que, los agricultores necesitan aplicar más fertilizante químico del que la planta necesita y el resto a menudo se libera al medio ambiente, contaminando el aire y el agua (Vance, 2001). Además, la producción industrial de fertilizantes químicos contribuye a las emisiones globales de $\mathrm{CO}_{2}$ (Vance, 2001).

La producción de chile habanero se realiza mayoritariamente con fertilización química (Reyes y Cortéz, 2017; Ramírez-Vargas et al., 2019), en diversas dosificaciones, dependiendo de los recursos económicos del productor y en muchas ocasiones sin el asesoramiento técnico requerido (Grageda-Cabrera et al., 2012).

Una forma en la que se puede reducir el uso de fertilizantes químicos sin dañar la nutrición de las plantas es mejorando la absorción de nutrientes por parte de los cultivos mediante el uso de bioestimulantes en la fertilización de las plantas (Halpern et al., 2015; Torres et al., 2016; Rodríguez-Hernández et al., 2020). Los bioestimulantes agrícolas son sustancias o microorganismos que se aplican a las plantas con el objetivo de mejorar la eficiencia nutricional, la tolerancia al estrés abiótico y la calidad del cultivo, siendo algunos de éstos bioestimulantes, productos comerciales que contienen una mezcla de sustancias y microorganismos (du Jardin, 2015). Los bioestimulantes microbianos incluyen hongos micorrízicos y no micorrízicos, bacterias endosimbióticas y rizobacterias promotoras del crecimiento vegetal (Calvo et al., 2014; Halpern et al., 2015).

El uso de bioestimulantes microbianos en la agricultura moderna ha aumentado considerablemente el interés por estudiarlos y conocer sus efectos (Calvo et al., 2014), se han evaluado como estimulantes de maíz y frijol (Hernández et al., 2017), como bioestimulantes en el crecimiento en plántulas de maracuyá (Díaz et al., 2020) y cultivos hortícolas bajo condiciones de estrés abiótico (Bulgari et al., 2019); así como promotores del crecimiento vegetal y manejo sostenible de nematodos fitoparásitos (D’Addabbo et al., 2019).

Además, se han evaluado en respuesta a las características agronómicas de plántulas y calidad del fruto en chile (Candelero et al., 2015; Gamboa-Angulo et al., 2020). Los bioestimulantes en la horticultura requieren ser evaluados local y temporalmente, se necesitan herramientas de la eficiencia de los bioestimulantes y de esta manera definir mecanismos que optimicen su uso. El objetivo de este trabajo fue evaluar tres bioestimulantes microbianos sobre la calidad de fruto y producción de chile habanero var. Jaguar bajo condiciones protegidas de macrotúnel.

\section{Materiales y métodos}

\section{Área de estudio}

El trabajo se realizó en el año 2021 en el Tecnológico Nacional de México, Campus Úrsulo Galván en las coordenadas de $19^{\circ} 24^{\prime} 43.12$ " latitud norte y $96^{\circ} 21$ ' 32.66" longitud oeste, ubicado en el municipio de Úrsulo Galván, en la región centro costera de Veracruz. El clima de esta región se 
clasifica como Aw (tropical húmedo-seco) por el sistema Köppen-Geiger, definido como cálido subhúmedo con lluvias en verano, con un rango de temperatura que oscila entre 24 y $26^{\circ} \mathrm{C}$ y un rango de precipitación entre 1100 y 1300 mm (INAP, 2013).

\section{Material vegetal}

Las semillas de chile habanero utilizadas fueron de la variedad Jaguar inoculadas con rhizophagus proporcionadas por el INIFAP Campo Experimental Cotaxtla. Las semillas fueron germinadas en charolas en el Campo Experimental Cotaxtla.

\section{Bioestimulantes}

Los bioestimulantes que se utilizaron fueron productos a base de Trichoderma spp., y bacterias fijadoras de nitrógeno Bacillus spp. Los tratamientos evaluados fueron: 1) T22; 2) Mix; 3) Genifix; y 4) testigo (Cuadro 1).

Cuadro 1. Tratamientos utilizados en la evaluación de los bioestimulantes en semillas y plantas de chile habanero ( $C$. chinense var. Jaguar).

\begin{tabular}{|c|c|c|c|}
\hline Tratamientos & Ingrediente activo & Compañía & Dosis \\
\hline $\begin{array}{c}\mathrm{T} 22^{\circledR}+\text { micorriza } \\
\text { INIFAP }^{\circledR}\end{array}$ & Trichoderma harzianum + micorriza & PHC e INIFAP & $0.5 \%(\mathrm{p} / \mathrm{v})$ \\
\hline MIX $^{\circledR}$ & $\begin{array}{c}\text { T. harzianum, } T \text {. viride, } T \text {. asperellum, } T . \\
\text { koningli }\end{array}$ & $\begin{array}{l}\text { Organismos } \\
\text { benéficos }\end{array}$ & $0.5 \%(\mathrm{p} / \mathrm{v})$ \\
\hline Genifix $^{\circledR}$ & $\begin{array}{l}\text { Bacillus sp. JVN5, B. megaterium strain } \\
\text { VVM1, Bacillus sp. FDMC4, B. subtilis } \\
\text { strain JAG3, B. megaterium strain EAV2 }\end{array}$ & $\begin{array}{l}\text { TecNM Campus } \\
\text { Úrsulo Galván }\end{array}$ & $20 \%(\mathrm{v} / \mathrm{v})$ \\
\hline Testigo & Agua & & \\
\hline
\end{tabular}

Evaluación de bioestimulantes en semillas de chile habanero. En charolas con sustrato Peat Moss se inocularon 100 semillas de chile habanero variedad Jaguar con cada uno de los tratamientos. Para los tratamientos 1 y 2, se aplicaron $10 \mathrm{ml}$ de T22 y Mix, respectivamente. El tratamiento 3 se inoculó con $1 \mathrm{ml}$ de Genifix. Las variables de respuesta fueron plántulas germinadas, altura de plántula a los 20 días después de la inoculación y peso seco de plántulas $\left(65^{\circ} \mathrm{C}\right.$ durante $\left.72 \mathrm{~h}\right)$ a los 20 días de la inoculación.

Evaluación de bioestimulantes en plantas de chile habanero. Se utilizó un macrotúnel de $3 \mathrm{~m}$ de ancho por $30 \mathrm{~m}$ de largo, forrado con malla antiáfidos. Dentro del macrotúnel se construyeron dos camas con composta mezclada con suelo y acolchado blanco-negro, las camas fueron de $90 \mathrm{~cm}$ de ancho y $30 \mathrm{~cm}$ de altura, separadas una de otra por un callejón de no menos de $40 \mathrm{~cm}$ de ancho, el marco de plantación fue de una planta cada $25 \mathrm{~cm}$, lo cual dio un total de 120 plantas por cama y 240 por macrotúnel. Se utilizó un sistema de riego de cuatro salidas de agua y $30 \mathrm{~m}$ de cintilla calibre 6000 para cada cama, conectadas a la línea principal con cuatro válvulas de paso para controlar el riego del cultivo. El diseño experimental fue en bloques completos al azar con cuatro repeticiones. En cada bloque experimental los bioestimulantes se aplicaron mensualmente al suelo, dirigidos al cuello de la planta (drench). 
El manejo del cultivo consistió en la aplicación de ácidos húmicos (10\%) a los 15 días después del trasplante (DDT); a través, del sistema de riego, a los 20, 50, 90 y 120 DDT se aplicaron fertilización química en drench, $20 \mathrm{ml}$ por planta y aplicaciones foliares de micronutrientes. A los 80 DDT se aplicó boro/calcio (Cuadro 2). A los 115 DDT se aplicó el abono foliar Bayfolan ${ }^{\circledR}$ $(0.2 \%)$ para la inducción de floración.

Cuadro 2. Fertilización química aplicada al suelo dirigido al cuello de la planta.

\begin{tabular}{|c|c|c|}
\hline Ingrediente & Nombre comercial & Dosis \\
\hline Fósforo/nitrato & DAP + urea & $1 \mathrm{~g} \mathrm{DAP}+1 \mathrm{~g}$ urea en $20 \mathrm{ml}^{-1}$ \\
\hline Micronutrientes & PoliQuel & Foliar $2 \mathrm{~L} \mathrm{ha}^{-1}$ en $200 \mathrm{~L}$ de agua \\
\hline Boro/calcio & Boro/calcio & Foliar $2 \mathrm{~L} \mathrm{ha}^{-1}$ en $200 \mathrm{~L}$ de agua \\
\hline
\end{tabular}

Se utilizaron los frutos de cuatro cortes, a los 111, 118, 136 y 146 días. Las variables de respuesta fueron: altura y peso de plántula a los 20 días después de la inoculación, peso y calidad de fruto (diámetro ecuatorial y diámetro polar) y peso de 20 frutos elegidos al azar por tratamiento. Análisis estadístico. Para comparar el efecto de los bioestimulantes en la germinación de las semillas de chile habanero se realizó un análisis no paramétrico de Friedman y para comparar el efecto en altura de plántula, peso de plántula, peso y calidad de fruto se realizó un Anova y una comparación de medias de Tukey $\alpha=0.05$. Además, también se analizó la interacción corte + bioestimulante. Los análisis estadísticos se realizaron con el software InfoStat versión 2020.

\section{Resultados y discusión}

Las semillas de chile habanero tratadas con los bioestimulantes Genifix y Mix registraron 94\% de germinación a diferencia de las semillas tratadas con el bioestimulante T22 y el testigo, que sólo obtuvieron 90 y $87 \%$ de germinación respectivamente; sin embargo, estas diferencias no fueron significativas $\left(\mathrm{T}^{2}=1.16, p=0.3254\right)$ en el análisis estadístico de Friedman. En cuanto a la altura de las plántulas a los 20 días después de la inoculación, las semillas inoculadas con el bioestimulante Genifix fueron las que alcanzaron la mayor altura, fueron significativamente diferentes $\left(\mathrm{F}_{3,959}=\right.$ 405.97, $p=0.0001$ ) a las semillas inoculadas con los otros tratamientos (Cuadro 2).

Las semillas inoculadas con el bioestimulante Mix no registraron diferencias significativas en altura de la plántula con respecto al testigo (Cuadro 2). Para el peso seco de plántulas, a los 20 días después de inoculación, las semillas inoculadas con el bioestimulante Genifix, fueron las que lograron mayor peso de plántulas y fueron significativamente diferentes $\left(\mathrm{F}_{3,959}=18.75, p=0.0001\right)$ a las semillas tratadas con el bioestimulante T22 y el testigo (Cuadro 3).

Cuadro 3. Efecto de tres bioestimulantes en la altura y peso de plántula de chile habanero.

\begin{tabular}{ccc}
\hline Bioestimulantes & Altura $(\mathrm{cm})$ & Peso $(\mathrm{g})$ \\
\hline Genifix & $6.53 \pm 0.04 \mathrm{a}$ & $0.037 \pm 0.004 \mathrm{a}$ \\
T22 & $6.04 \pm 0.12 \mathrm{~b}$ & $0.029 \pm 0.001 \mathrm{c}$ \\
Mix & $3.82 \pm 0.12 \mathrm{c}$ & $0.035 \pm 0.001 \mathrm{ab}$ \\
Testigo & $3.68 \pm 0.08 \mathrm{c}$ & $0.032 \pm 0.004 \mathrm{bc}$ \\
CV $(\%)$ & 19.01 & 12.02 \\
\hline
\end{tabular}

Medias con una letra común no son significativamente diferentes $(p>0.05)$. 
Para la variable diámetro polar del fruto, las plantas tratadas con los bioestimulantes tuvieron frutos significativamente más grandes $\left(\mathrm{F}_{3,4028}=98.44, p=0.0001\right)$ que las plantas testigos (Cuadro 4). Al comparar únicamente a los bioestimulantes se observó que los frutos de las plantas tratadas con el bioestimulante T22 fueron en promedio más grandes a los frutos de las plantas tratadas con el bioestimulante Mix (Cuadro 4). El bioestimulante Genifix no presentó diferencias significativas en relación a los otros dos bioestimulantes (Cuadro 4).

Cuadro 4. Efecto de tres bioestimulantes en diámetro polar y diámetro ecuatorial de frutos de chile habanero.

\begin{tabular}{ccc}
\hline Bioestimulantes & Diámetro polar $(\mathrm{cm})$ & Diámetro ecuatorial $(\mathrm{cm})$ \\
\hline T22 & $4.13 \pm 0.03 \mathrm{a}$ & $3.12 \pm 0.02 \mathrm{ab}$ \\
Genifix & $4.03 \pm 0.02 \mathrm{ab}$ & $3.06 \pm 0.02 \mathrm{~b}$ \\
Mix & $3.99 \pm 0.02 \mathrm{~b}$ & $3.15 \pm 0.02 \mathrm{a}$ \\
Testigo & $3.53 \pm 0.03 \mathrm{c}$ & $2.82 \pm 0.02 \mathrm{c}$ \\
CV $(\%)$ & 8.75 & 7.49 \\
\hline
\end{tabular}

Medias con una letra común no son significativamente diferentes ( $p>0.05)$.

Al analizar la interacción corte + bioestimulante, se encontró que los primeros tres cortes + cualquiera de los bioestimulantes resultaron con frutos significativamente más grandes $\left(\mathrm{F}_{9}, 4028=\right.$ $8.48, p=0.0001)$ que los testigos en cualquiera de los cortes. La mejor interacción que se dio fue el corte uno + el bioestimulante T22. En la variable diámetro ecuatorial de fruto se registraron diferencias significativas $\left(\mathrm{F}_{3}, 4028=66.82, p=0.0001\right)$ entre los tratamientos; sin embargo, los resultados se invirtieron en cuanto a los bioestimulantes, los frutos de las plantas tratadas con el bioestimulante Mix fueron los que presentaron un diámetro ecuatorial mayor en comparación con los frutos de las plantas tratadas con el bioestimulante Genifix (Cuadro 4).

El estimulante T22 no obtuvo diferencias estadísticas en relación con los otros dos bioestimulantes (Cuadro 4). Al comparar los bioestimulantes con el testigo, los resultados fueron similares al de diámetro polar de fruto, ya que las plantas tratadas con cualquiera de los bioestimulantes tuvieron frutos más anchos que los que se obtuvieron en las plantas testigo (Cuadro 4). En cuanto a la interacción, el corte dos + el bioestimulante Mix fue la que generó frutos más anchos y fue significativamente diferente $\left(\mathrm{F}_{9,4028}=9.18, p=0.0001\right)$ a todas las interacciones corte + testigo.

En cuanto a la variable peso por fruto, las plantas tratadas con los bioestimulantes produjeron frutos significativamente más pesados $\left(\mathrm{F}_{3}, 4028=69.43, p=0.0001\right)$ que las plantas testigo y no se obtuvieron diferencias estadísticas entre los bioestimulantes (Cuadro 5). Las interacciones de los cortes uno, dos y tres + cualquier bioestimulante registraron frutos significativamente más pesados $\left(\mathrm{F}_{9,4028}=2.25, p=0.0169\right)$ que las interacciones que incluían cualquier corte + el testigo.

Para el peso de muestra de 20 frutos, las plantas tratadas con los bioestimulantes Genifix y Mix registraron los mayores pesos de muestra y fueron significativamente diferentes $\left(\mathrm{F}_{3,819}=17.90, p=\right.$ 0.0001) al testigo (Cuadro 5). En cuanto a la interacción, el corte tres + el bioestimulante Genifix o Mix fueron significativamente diferentes $\left(\mathrm{F}_{9,819}=3.32, p=0.0005\right)$, con el mayor peso de muestra de 20 frutos, a los testigos en los cuatro cortes. También la interacción corte cuatro + bioestimulante T22, tuvo mayor peso de muestra de 20 frutos que los testigos en cualquiera de los cortes. 
Cuadro 5. Efecto de tres bioestimulantes en peso por fruto y peso total de frutos de chile habanero.

\begin{tabular}{ccc}
\hline Bioestimulantes & Peso $(\mathrm{g}) \mathrm{x}$ fruto & Peso $(\mathrm{g}) \mathrm{x} 20$ frutos \\
\hline T22 & $10.18 \pm 0.11 \mathrm{a}$ & $300.74 \pm 13.45 \mathrm{a}$ \\
Mix & $10.07 \pm 0.1 \mathrm{a}$ & $305.38 \pm 14.44 \mathrm{a}$ \\
Genifix & $9.98 \pm 0.1 \mathrm{a}$ & $303.75 \pm 13.11 \mathrm{a}$ \\
Testigo & $8.27 \pm 0.12 \mathrm{~b}$ & $186.83 \pm 13.49 \mathrm{~b}$ \\
CV $(\%)$ & 15.46 & 34.5 \\
\hline
\end{tabular}

Medias con una letra común no son significativamente diferentes ( $p>0.05)$.

Los resultados corroboran lo reportado en otras investigaciones sobre los efectos positivos de los bioestimulantes a base de Trichoderma spp. y Bacillus spp. En hortalizas (Diánez et al., 2018; Gamboa-Angulo et al., 2020; Rojas-Badía et al., 2020). Sin embargo, en la germinación de chile habanero, los resultados indicaron un nulo efecto de los bioestimulantes, lo cual puede deberse a que algunas cepas de Trichoderma o Bacillus disminuyan o no tengan un efecto estimulante en la germinación de chile habanero como lo reporta Sosa-Pech et al. (2019) para aislados de Bacillus CBCC57 y CBFRF5, los cuales obtuvieron una menor germinación en relación con el testigo.

También se ha reportado variaciones del efecto de cepas de Bacillus en la germinación de pimiento, donde se indica que la aplicación de Bacillus sp. MA06 aumentó el porcentaje de germinación en un $8 \%$, pero tres cepas de Bacillus no mostraron diferencia significativa con relación a las semillas sin inocular (Luna et al., 2013).

Por otro lado, Ezziyyani et al. (2004) obtuvieron sólo un $60 \%$ de germinación en semillas de pimiento tratadas con $T$. harzianum a los 10 días. Sin embargo, a diferencia de estos resultados, también se han reportado efectos positivos en la germinación de semillas de chile tratadas con $T$. harzianum con porcentajes de germinación de 82 y 90.3\% (Madhavi et al., 2006; Miguel-Ferrer et $a l ., 2021)$ y en cuanto a bacterias del género Bacillus, Kaymak et al. (2009), indican que $B$. megaterium mejoró el porcentaje y la tasa de germinación en semillas de rábano.

También en esta investigación se demostró con estos resultados que el bioestimulante Genifix, a base de bacterias Bacillus, tiene un efecto significativo en el desarrollo de plántulas de chile habanero, esto debido a que Bacterias del género Bacillus promueven y estimulan el crecimiento vegetal a través de la síntesis de hormonas en la planta, como citocininas, etileno y giberelinas (Rojas-Solís et al., 2013); así como a través, de la fijación de nitrógeno y fósforo (Corrales et al., 2017; Rodríguez-Hernández et al., 2020).

Asimismo, Sosa-Pech et al. (2019) han reportado que aislados de Bacillus promueven el crecimiento a nivel de plántula de chile habanero, entre sus tratamientos, los aislados CBCC57 y CBRF12 promovieron el crecimiento en altura de planta y área foliar. A demás, Kokalis-Burelle et al. (2002) indicaron que la formulación LS256 (Bacillus subtilis GBO3 y B. pumilis INR7) promovió el crecimiento del tallo, raíz y parte aérea en plántulas de chile. Para Trichoderma, Candelero et al. (2015) reportan cepas que mejoraron significativamente la altura de plántulas, longitud de raíz, volumen radical y biomasa seca total de Capsicum chinense, lo que coincide con los resultados obtenidos del bioestimulante T22 en altura de plántula. 
En cuanto a la calidad del fruto, los resultados demostraron que los bioestimulantes mejoraron significativamente el tamaño y peso del fruto de chile habanero en comparación al testigo, lo que complementa la información generada por Gamboa-Angulo et al. (2020) sobre los efectos positivos en la calidad interna de frutos de chile en relación con el contenido de lípidos, proteínas y fósforo, cuando las plantas son biofertilizadas con T. harzanium y B. subtilis. Se tiene poca información de los efectos de bioestimulantes en las dimensiones y peso de frutos, lo cual es necesario establecer y relacionar con características nutrimentales. Se han evaluado inoculantes microbianos a base de Rhizophagus irregularis, Pseudomonas spp. y Azospirillum brasilense en rendimiento y tamaño de fruto de chile habanero, reportándose que la inoculación de Pseudomonas spp. A chile habanero en el trasplante aumenta el crecimiento, rendimiento y tamaño de fruto (Reyes-Ramírez et al., 2014).

\section{Conclusiones}

Se demostró con los resultados que el bioestimulante Genifix tuvo mayor eficiencia para estimular el desarrollo de plántulas de chile habanero en comparación a los bioestimulantes T22 y Mix. El bioestimulante T22 mejoró significativamente la longitud de los frutos de chile habanero y el bioestimulante Mix el ancho de los frutos. Todos los bioestimulantes incrementaron significativamente el peso de fruto de chile habanero, sin presentar diferencias significativas entre ellos. Se requiere seguir evaluando bioestimulantes en la horticultura de manera local y temporal para dar seguimiento a la eficiencia de los productos y de esta manera definir mecanismos que optimicen su uso.

\section{Agradecimientos}

A los proyectos 'Diversidad genética de microorganismos de vida libre con potencial en fijación biológica de nitrógeno, como alternativa de biofertilización' clave 6218.19-P, 'Evaluación de bioestimulantes y bioinsecticidas en condiciones de macrotúnel en la producción de hortalizas' clave 10544.21-P y 'Sistema de producción biorracional de hortalizas en macrotúnel, conducido por mujeres' clave 142252 , por el financiamiento para los trabajos.

\section{Literatura citada}

Bulgari, R.; Franzoni, G. and Ferrante, A. 2019. Biostimulants application in horticultural crops under abiotic stress conditions. Agronomy. 9(306):1-30. https://doi.org/10.3390/agronomy 9060306.

Calvo, P.; Nelson, L. and Kloepper, J. W. 2014. Agricultural uses of plant biostimulants. Plant Soil. 383(2014):3-41.

Candelero, D. J.; Cristóbal, A. J.; Reyes, R. A.; Tun, S. J. M.; Gamboa, A. M. M. y Ruíz, S. E. 2015. Trichoderma spp. promotoras del crecimiento en plántulas de Capsicum chinense Jacq. y antagónicas contra Meloidogyne incognita. ФYTON. 84(1):113-119.

Corrales, R. L. C.; Caycedo, L. L.; Gómez, M. M. A.; Ramos, R. S. J. y Rodríguez, T. J. N. 2017. Bacillus spp: una alternativa para la promoción vegetal por dos caminos enzimáticos. Nova. 15(27):45-65. https://doi.org/10.22490/24629448.1958.

Du-Jardin, P. 2015. Plant biostimulants: definition, concept, main categories, and regulation. Sci. Hortic. 196(2015):3-14. http://dx,doi.org/10.1016/j.scienta.2015.09.021. 
D’Addabbo, T.; Laquale, S.; Perniola, M. and Candido, V. 2019. Biostimulants for plant growth promotion and sustainable management of phytoparasitic nematodes in vegetable crops. Agronomy. 9(616):1-10. https://doi.org/10.3390/agronomy9100616.

Daverede, I. C.; Kravchenko, A. N.; Hoeft, R. G.; Nafziger, E. D.; Bullock, D. G.; Warren, J. J. and Gonzini, L. C. 2004. Phosphorus runoff from incorporated and surface-applied liquid swine manure and phosphorus fertilizer. J. Environ. Quality. 33(4):1535-1544. https://doi.org/10.2134/jeq2004.1535.

Diánez, F.; Santos, M.; Carretero, F. and Marín, F. 2018. Biostimulant activity of trichoderma saturnisporum in melon (Cucumis melo). HortScience. 53(6):810-815. https://doi.org/ 10.21273/hortsci13006-18.

Díaz, G.; Rodríguez, G.; Montana, L.; Miranda, T.; Basso, C. y Arcia, M. 2020. Efecto de la aplicación de bioestimulantes y Trichoderma sobre el crecimiento en plántulas de maracuyá (Passiflora edulis Sims) en vivero. Bioagro. 32(3):195-204. https://revistas.uclave.org/ index.php/bioagro/article/view/2787.

Ezziyyani, M.; Sánchez, C. P.; Ahmed, A. S.; Requena, M. E. y Castillo, M. E. C. 2004. Trichoderma harzianum como biofungicida para el biocontrol de Phytophthora capsici en plantas de pimiento (Capsicum annuum L.). Anales de Biología. 26:35-45. https://revistas.um.es/analesbio/article/view/30441.

Gamboa-Angulo, J.; Ruíz-Sánchez, E.; Alvarado-López, C.; Gutiérrez-Miceli, F.; RuízValdiviezo, V. M. y Medina-Dzul, K. 2020. Efecto de biofertilizantes microbianos en las características agronómicas de la planta y calidad del fruto del chile xcat'ik (Capsicum annuиm L.). Terra Latinoam. 38(4):817-826. https://doi.org/10.28940/terra.v38i4.716.

Grageda-Cabrera, O. A.; Díaz-Franco, A.; Peña-Cabriales, J. J. y Vera-Núñez, J. A. 2012. Impacto de los biofertilizantes en la agricultura. Rev. Mex. Cienc. Agríc. 3(6):1261-1274.

Halpern, M.; Bar-Tal, A.; Ofek, M.; Minz, D.; Muller, T. and Yermiyahu, U. 2015. The use of biostimulants for enhancing nutrient uptake. In: advances in agronomy. Sparks, D. L. (Ed.). Vol. 129. Elsevier Inc. Netherlands. 141-174 pp. https://doi.org/10.1016/bs.agron. 2014.10.001.

Hernández, M. S.; Novo, S. R.; Mesa, P. M. A.; Ibarra, M. A. y Hernández, R. D. 2017. Capacidad de Trichoderma spp. como estimulante de la germinación en maíz (Zea mays L.) y frijol (Phaseolus vulgaris L.). Rev. Gest. Con. Des. Loc. 4(1):19-23.

Kaymak, H. C.; Guvenc, I.; Yarali, F. and Donmez, M. F. 2009. The effects of bio-priming with PGPR on germination of radish (Raphanus sativus L.) seeds under saline conditions. Turkish J. Agric. Fores. 33(2):173-179. https://doi:10.3906/tar-0806-30.

Kokalis-Burelle, K.; Vavrina, C. S.; Rosskopf, E. N. and Shelby, R. A. 2002. Field evaluation of plant growth-promoting rhizobacteria amended transplant mixes and soil solarization for tomato and pepper production in Florida. Plant and Soil. 238:257-266. https://doi.org/10.1023/A:1014464716261.

Luna, M. L.; Martínez, P. R. A.; Hernández, I. M.; Arvizu, M. S. M. y Pacheco, A. J. R. 2013. Caracterización de rizobacterias aisladas de tomate y su efecto en el crecimiento de tomate y pimiento. Rev. Fitotec. Mex. 36(1):63-69.

Madhavi, M.; Kumar, C. P. C.; Reddy, D. R. R. and Singht, T. K. 2006. Integrated management of wilt of chilli incited by Fusarium solani. Ind. J. Plant Protec. 34(2):225-228.

Miguel-Ferrer, L.; Romero-Arenas, O.; Andrade-Hoyos, P.; Sánchez-Morales, P. and RiveraTapia, J. A. 2021. Antifungal activity of Trichoderma harzianum and T. koningiopsis against Fusarium solani in seed germination and vigor of Miahuateco chili seedlings. Rev. Mex Fitopatol. 39(2):228-247. 
Ramírez-Vargas, B. A.; Carrillo-Ávila, E.; Obrador-Olán, J. J.; Coh-Méndez, D.; MonsalvoEspinosa, A. y Aceves-Navarro, E. 2019. Aplicación del modelo simplificado para estimar dosis sustentables de fertilización fosforada en el cultivo de chile habanero (Capsicum chinense Jacq.). Investigación y Ciencia. 27(78):23-33.

Reyes, G. y Cortéz, D. 2017. Intensidad en el uso de fertilizantes en América Latina y el Caribe (2006-2012). Bioagro. 29(1): 45-52.

Reyes-Ramírez, A.; López-Arcos, M.; Ruiz-Sánchez, E.; Latournerie-Moreno, L.; Pérez-Gutiérrez, A.; Lozano-Contreras, M. G. y Zavala-León, M. J. 2014. Efectividad de inoculantes microbianos en el crecimiento y productividad de chile habanero (Capsicum chinense Jacq.). Agrociencia. 48(3):285-294.

Rodríguez-Hernández, M. G.; Gallegos-Robles, M. Á.; Rodríguez-Sifuentes, L.; Fortis-Hernández, M.; Luna-Ortega, J. G. y González-Salas, U. 2020. Cepas nativas de Bacillus spp. como una alternativa sostenible en el rendimiento de forraje de maíz. Terra Latinoam. 38(2):313321. https://doi.org/10.28940/terra.v38i2.690.

Rojas-Badía, M. M.; Bello-González, M. A.; Ríos-Rocafull, Y.; Lugo-Moya, D. y Rodríguez, S. J. 2020. Utilización de cepas de Bacillus como promotores de crecimiento en hortalizas comerciales. Acta Agron. 69(1):54-60. https://doi.org/10.15446/acag.v69n1.79606.

Rojas-Solís, D.; Contreras-Pérez, M. y Santoyo, G. 2013. Mecanismos de estimulación del crecimiento vegetal en bacterias del género Bacillus. Biológicas. 15(2):36-41.

Sánchez, L.; Diez, J. A.; Vallejo, A. and Cartagena, M. C., 2001. Denitrification losses from irrigated crops in central Spain. Soil Biol. Beachem. 33(9):1201-1209. https://doi.org/ 10.1016/S0038-0717(01)00024-4.

Sosa-Pech, M.; Ruiz-Sánchez, E.; Tun-Suárez, J. M.; Pinzón-López, L. L. y Reyes-Ramírez, A. 2019. Germinación, crecimiento y producción de glucanasas en Capsicum chinense Jacq. Inoculadas con Bacillus spp. Ecosistemas y Recursos Agropecuarios. 6(16):137-143. https://doi.org/10.19136/era.a6n16.1801.

Torres, R. J. A.; Reyes, P. J. J. y González, R. J. C. 2016. Efecto de un bioestimulante natural sobre algunos parámetros de calidad en plántulas de tomate (Solanum lycopersicum, L.) bajo condiciones de salinidad. Biotecnia. 18(2):11-15. https://doi.org/10.18633/bt.v18i2.274.

Vance, C. P. 2001. Symbiotic nitrogen fixation and phosphorus acquisition. Plant nutrition in a world of declining renewable resources. Plant Physiol. 127(2):390-397. https://doi.org/10.1104/pp.010331. 